\title{
Top Ten Tips Palliative Care Clinicians Should Know About Integrating Population Health Principles into Practice
}

\author{
Devon K. Check, PhD, ${ }^{1,4}$ Brystana G. Kaufman, PhD, ${ }^{2}$ Arif H. Kamal, MD, MHS, MBA, ,3,4 \\ and David J. Casarett, MD, MA ${ }^{3,4}$
}

\begin{abstract}
The field of palliative care (PC) has spent the past decade demonstrating that it improves outcomes for patients, clinicians, and health systems. Forward-thinking organizations preparing for a reimbursement system rooted in value have built robust inpatient PC programs and are rapidly moving toward the outpatient and community settings as well. As PC programs get larger and are increasingly tasked with leading a wide variety of diverse initiatives, population health principles can help to focus programs on high-value activities. This article, written by population health researchers and PC clinicians, seeks to provide PC teams nationally with a variety of population health strategies and tools to guide PC delivery throughout the health system and beyond.
\end{abstract}

Keywords: clinical decision support tools; palliative care; patient-facing interventions; population-based approach; population health; serious illness

\section{Introduction}

Pecialty palliative CARE (PC) services have grown nearly $150 \%$ in the past decade. ${ }^{1}$ Despite such remarkable growth, a shortage of PC specialists limits access and necessitates standardized approaches to match PC services with patients who may benefit the most. ${ }^{2} \mathrm{PC}$ programs can close gaps in the accessibility of PC for patients with serious illness by incorporating principles of population health. Population health focuses on improving the health of defined populations, with special emphasis on promoting equity in care delivery. A population-based approach to PC delivery strives to meet the needs of all patients and families facing serious illness by (1) enhancing access to and promoting the use of specialized PC services for those would most benefit from them and (2) extending the "ingredients" of PC beyond specialized PC consultations.

Based on our collective experience as population health researchers (D.K.C. and B.G.K.) and leaders within clinical PC (A.H.K. and D.J.C.), we discuss 10 ways that PC programs can integrate strategies and tools of populationbased PC delivery into their practices and surrounding communities.

\section{Tip 1: Data-Informed Approaches Are Key to Proactively Identifying Patients Who Could Benefit from PC Consultations}

Population health programs leverage available data to identify patients who make up the target population. Datainformed tools for defining a target population typically collect health information about individuals from electronic health records (EHRs) and/or insurance claims databases. ${ }^{3,4}$ Novel methods to stratify patients by risk and identify actionable populations are being developed, spurred by the analytical power of modern computers (e.g., deep networks) and growth of alternative payment models. ${ }^{5,6}$ Once formed, such tools allow a population management program to proactively take steps to improve population members' care and to systematically track care processes and outcomes. ${ }^{7}$

A unique challenge for PC is the lack of widely accepted criteria for identifying patients who would benefit from services. However, patients with limited prognosis, high health care utilization, poorly controlled symptoms, or worsening functional status represent populations for whom PC interventions can have a positive impact. ${ }^{4}$ For example, health systems can leverage the EHR to create patient lists based on

Departments of ${ }^{1}$ Population Health Sciences and ${ }^{3}$ Medicine, Duke University Medical Center, Durham, North Carolina.

${ }^{2}$ Margolis Center for Health Policy, Duke University, Durham, North Carolina.

${ }^{4}$ Duke Cancer Institute, Duke University Medical Center, Durham, North Carolina.

Accepted February 20, 2020. 
select diagnoses known to have a limited prognosis and/or high symptom burden. Clinical events such as hospitalizationswhich, for some conditions, can represent an inflection point in the disease trajectory - may also serve as indicators of need for a PC consultation. ${ }^{4}$ Further, decrements in functional status, reflected in clinical documentation (e.g., performance status scales), may highlight patients who could benefit from PC.

More complex approaches may involve linkage across data sources. For example, the PC registry developed by Kaiser Permanente Northern California-a fully integrated health care financing and delivery system-leverages clinical data (e.g., diagnoses and lab values) from the EMR in combination with hospital and durable medical equipment claims (e.g., for in-home oxygen) to identify patients potentially in need of a PC consultation. ${ }^{8}$ Programs may also consider approaches that combine use of clinical information from the EHR with providers' validation of appropriateness for PC, for example, using the validated "surprise" question."

\section{Tip 2: Patient-Facing Interventions May Help} to Improve Patients' Willingness to Engage with PC

Identifying and facilitating referrals for patients who may benefit from PC are necessary but likely not sufficient for promoting patient engagement in consultations. Even in settings with robust PC programs, it is estimated that only $40 \%$ of patients who could benefit receive any specialty services. ${ }^{10}$ Ongoing misperceptions among patients and family members regarding the value of PC continue to impede uptake. For example, one study evaluating PC knowledge and understanding among 152 laypeople found that most could answer less than half of knowledge questions correctly. ${ }^{11}$ Another recent study found that nearly three-quarters of U.S. adults had never heard of PC. ${ }^{12}$ As a result, no-show rates in PC clinics can approach $40 \% .^{13}$

Though evidence-based interventions specifically targeting PC literacy are few, PC programs could consider adopting available educational tools. Video-based decision aids (e.g., advance care planning [ACP] decisions) ${ }^{14}$ and ACP education resources (e.g, PREPARE for your care) ${ }^{15}$ have demonstrated in studies the ability to increase patient knowledge and engagement. ${ }^{16,17}$ In addition, PCforMe, a theory-driven, patient-facing mobile health tool, was recently developed by our team to improve understanding of PC and reduce misunderstanding among patients referred for consultations. PCforMe presents video vignettes on a PC-related topic, and then it asks users to apply newly acquired knowledge to their own situation in a short activity. Preliminary results support the tool's usability and potential for increasing participants' knowledge of PC and decreasing PC clinics' no-show rates. ${ }^{13}$

\section{Tip 3: PC Teams Should Leverage Social Marketing Programs to Bring Conversations About Serious IIIness and End-of-Life Issues into the Mainstream}

To promote the use of PC among patients and families, PC programs should create strategies and tactics to contribute to changes in existing cultural paradigms around serious illness and end-of-life care. ${ }^{18}$ A key step toward changing culture is encouraging increased openness about serious illness and death. PC programs should consider promoting existing models of community engagement within their health systems and local communities. For example, the "Before
I Die" participatory art project invites community members to contemplate death and reflect upon their lives, with the goal of destigmatizing death. The program-which spans 75 countries and 35 languages - offers a toolkit for organizations and communities who wish to participate. ${ }^{19}$ In addition, in 2013, Australia adopted a public health initiative_-'Dying to Know" - which designates an annual day of action dedicated to promoting conversations and community actions around death, dying, and bereavement. Dying to Know Day events include will writing and ACP workshops, group visits to funeral homes to discuss "after death" options, legacy leaving sessions, and death cafes. ${ }^{20}$

Individual clinicians can also play in a role in raising public awareness about end-of-life issues and PC. For example, the George Washington Cancer Center has developed a Palliative Care Awareness Social Media Toolkit to support professionals in implementing evidence-based strategies for communicating about $\mathrm{PC}$ via social media platforms. ${ }^{21}$

\section{Tip 4: A Population-Based PC Delivery System Relies on Three Groups of Professionals: PC Specialists, Clinicians Who Do not Specialize in PC but Provide Basic PC Services (i.e., Primary PC Clinicians), and Interprofessional Health Care Workers with Nonclinical Training}

A population-based approach to PC delivery must offer strategies to meet patients' and families' needs without relying solely on the services of PC specialists. One novel approach involves equipping clinicians from other specialties (e.g., primary care, oncology, cardiology) with the knowledge and skills needed to deliver a basic level of PC, including symptom management, emotional support, and care planning (i.e., primary PC education). ${ }^{4}$ Additional strategies ease the burden on clinical staff by engaging inter-professional health care workers, for example, care managers or patient navigators, to provide transitional care and care coordination services.

Care managers, who serve as patient advocates, work directly with patients across multiple treatment settings and payment sources. Patient navigators-who may or may not have a clinical background-aim at ensuring timely access to needed health care services by guiding patients through the increasingly complex health care system and helping them to overcome barriers to care. ${ }^{2,23}$ The flexible longitudinal relationships that care managers and patient navigators have with patients and caregivers uniquely position members of this workforce as sources of support for navigating serious illness.

In the following Tips 5-8, we discuss examples of how PC programs can facilitate the transfer of PC knowledge and skills from specialist to non-specialist clinicians and leverage care managers and patient navigators to facilitate advance care planning specifically to expand the scale of PC services delivered to a population.

\section{Tip 5: Non-specialist Clinicians Can Play a Critical Role in Primary PC Education and in Facilitating Effective Goals-of-Care Conversations}

Patients who engage in effective goals-of-care conversations with their clinicians receive fewer non-beneficial medical interventions and experience better quality of life. ${ }^{24-27}$ Yet, in practice, fewer than one-third of patients with serious illness report discussing their goals of care with their 
clinicians. $^{28}$ A known barrier to effective goals of care communication is clinicians' lack of confidence in initiating and navigating difficult conversations about end-of-life issues. ${ }^{29}$ In some settings, clinicians without PC training may be able to engage PC specialists to help facilitate goals-ofcare conversations. However, given the constraints of the PC workforce, it is critical that all clinicians who care for patients facing serious illness develop the skills needed to communicate effectively with patients about their goals.

Fortunately, goals-of-care communication skills training programs have emerged to address this need. VitalTalk, ${ }^{30}$ for example, is a widely adopted, evidence-based program that employs a train-the-trainer approach to improving communication skills. While originally developed for and evaluated among physicians (e.g., oncologists), VitalTalk is also relevant for advanced practice providers who care for patients with serious illness. For example, members of our team are currently conducting a pilot evaluation of VitalTalk training for nurse practitioners and physician assistants in the Duke Heart Center. Other communication training programs using the SPIKES ${ }^{31}$ and Best Case/Worst Case ${ }^{32}$ frameworks have also demonstrated promise in improving the communication skills of primary PC clinicians.

\section{Tip 6: Clinical Decision Support Tools Can Help to Further the Integration of Specialist Expertise with Primary PC}

Clinical decision support (CDS) tools are used to guide evidence-based, guideline-concordant care. ${ }^{7}$ In particular, EHRs can be designed to include "best practice alerts" for providers, flagging potential concerns and offering evidencebased guidance for responding to those concerns. In the population-based PC delivery context, CDS tools are being leveraged to support clinicians who are not PC specialists in effectively managing the symptoms of their seriously ill patients. For example, in oncology, evidence-based electronic patient-reported outcomes (PROs) systems that routinely monitor patients' symptoms are increasingly coupled with CDS to enable oncology providers to respond to patients' poorly controlled symptoms. Such CDS tools are developed based on clinical practice guidelines for further assessment, pharmacological and non-pharmacological interventions, and appropriate referrals. ${ }^{33}$

PC providers' expertise will be critical in developing similar PRO-enabled CDS tools for serious illness settings outside of oncology, where prevalence and severity of symptoms may be underrecognized (e.g., pain in chronic pulmonary disease $^{34}$ ) or the disease trajectory is less clear. For example, an EHR alert created with the PC team could suggest to liver specialists a PC consultation when an advanced liver disease patient is listed for transplant. This approach helps operationalize a common mantra in PC to "hope for the best but prepare for the worst.",

\section{Tip 7: PC Champions-Who Sit at the Nexus between Specialty PC and the Larger Clinical Workforce- Are Well Positioned to Provide Basic PC Services}

A PC champion is a clinician who is interested in PC, has been trained to skillfully assess and manage patients' $\mathrm{PC}$ needs, and promotes the learning and use of PC skills (e.g., communication) as part of standard practice within their own specialty. $^{35}$ In a population-based PC delivery system, champions can play a critical role in closing gaps in care for patients with serious illness.

$\mathrm{PC}$ champions require formal training in $\mathrm{PC}$, not necessarily through a fellowship, which may take the form of intensive training courses or master's programs. In addition, formal relationships with PC specialists - internal or external to the champion's home institution-are crucial for facilitating champions' access to support when faced with unfamiliar clinical issues and to supporting their continued development through regular case discussions. Equipped with training and ongoing support from PC specialist mentors, champions can fulfill the role of a PC expert in settings where a specialist team may not be available. In settings where specialist teams are available, leaders of those teams are well positioned to identify and support the development of PC champions, who can then serve as embedded PC advocates and promote basic PC education and quality improvement in their own specialties. ${ }^{35}$

\section{Tip 8: By Working with Patient Navigators, PC Programs Can Extend the Reach of Advance Care Planning}

Case management and patient navigation programs within health systems and insurance companies are growing due to the proliferation of Alternative Payment Models that align incentives with patient-centered care. The use of nonclinical personnel allows for a low-cost expansion of the work force available to support patients with a high risk of death, high symptom burden, and/or significant psychosocial dysfunction. In particular, trained lay navigators and care managers can use their lived experience to connect with patients and guide ACP conversations. Humana, for example, recently launched a pilot of care manager-facilitated structured ACP discussions among individuals with complex chronic conditions, including cancer. The Patient Care Connect Program and the Conversation Project are patient navigatorled ACP programs implemented by health systems in Alabama and Wisconsin, respectively. ${ }^{36,37}$

Care manager and navigator roles are tailored based on the patient population, health system or insurer goals, and resources available. As a result, there is significant variability in their parameters, including scope of practice. Regardless of the specific model, care managers and navigators are most successful when they are provided with evidence-based training and tools. Examples of such resources include the Case Managers Advance Care Planning Practices Instrument, Let Me Decide advance directive training program, and the Respecting Choices ${ }^{\circledR}$ First Steps ACP Facilitator training. $38-40$

\section{Tip 9: Population-Based PC Must Find Ways to Reach Patients Beyond the Health Care System, for Example, by Equipping Patients and Caregivers with the Knowledge and Skills Needed to Manage Symptoms at Home}

Patients are living longer with serious illness, and their care is moving out of the hospital, and into community-based settings, including patients' own residences. In fact, maximizing time at the place they call home is consistent with most seriously ill patients' goals and preferences. ${ }^{41}$ Given this reality, it 
is important to extend the reach of PC not only beyond specialist consultations but also beyond the health care system.

Receiving care at home-although in line with the goals of most patients - is associated with less adequate symptom control. $^{42}$ Further, poorly controlled symptoms, including pain, are commonly responsible for seriously ill patients' emergency department visits. ${ }^{43}$ Interventions that target patients' and caregivers' symptom management knowledge and support self-management behaviors are critical for improving quality of life at home. ${ }^{44}$

Fortunately, evidence-based approaches to support selfmanagement of symptoms exist, and PC programs should consider adopting them to support patients' and caregivers' ability to effectively manage symptoms at home. ${ }^{42,45}$ Most interventions have been developed and tested in the setting of pain related to cancer and share several key components. First, they are individualized to patients' specific needs, concerns, gaps in knowledge, and cultural context. Second, they address patients' knowledge, skills, and attitudes toward pain and its management, for example, by providing information about pain medications and their effects, and nonpharmacological pain management techniques. Other strategies include coaching sessions to help patients take their medications at the recommended intervals.

Third, they enable patients by engaging them in their own pain management, for example, by improving communication with clinicians and encouraging patients and caregivers to actively participate in decision making around pain management. Specific enablement tools include question prompt lists, personalized treatment plans, and instructions on how and when to contact clinicians about uncontrolled pain. Existing interventions vary widely in terms of their format, duration, and intensity, allowing PC programs the flexibility to identify interventions that may fit within their local contexts. With respect to timing, available data suggest that the optimal time to introduce self-management interventions may be the transition from inpatient hospital settings to home. ${ }^{42}$

\section{Tip 10: Mobile Apps for Advance Care Planning Can Facilitate Education About and Completion of ACP Outside of the Clinician-Patient Encounter}

For patients receiving care at home, mobile apps for ACP represent a useful and scalable tool for facilitating communication about end-of-life wishes between patients and their family members and completion of advance care directives. Some apps-for example, Everplans ${ }^{46}$ and MyDirectives ${ }^{47}$ serve as a digital archive for users' completed documents and/ or treatment goals. Other apps-such as Cake ${ }^{48}$ - help users to elucidate their goals and values. Once goals are identified, Cake offers action steps for ensuring that those goals are carried out. Even among patients who do engage in ACP as part of their encounters with PC or other clinicians, ACP apps can enhance preparedness to discuss ACP during the visit and facilitate the process, potentially allowing patients/providers to discuss and address patients' other PC needs (e.g., symptom management, spiritual concerns, caregiver support).

PC programs and individual PC providers should consider leveraging these and other mobile-based tools to supplement their own ACP conversations with patients. They may also champion wider use of these tools in health systems, for example among primary care and other specialist providers who care for seriously ill patients.

\section{Conclusion}

A population-based approach to PC delivery strives to promote the use of specialized PC services among those who need them and extend access to the "ingredients" of PC beyond specialized PC consultations. Enhancing access and uptake of PC consultations will require not only prospectively identifying and referring patients likely to benefit from PC but also providing those patients with educational interventions, and leading broader efforts to bolster communities' awareness and acceptance of PC. In addition, PC specialists can extend the reach of PC by helping to bridge the gap between specialist services and the broader health care workforce, primarily through training, mentorship, and CDS. Finally, with increasing numbers of seriously ill patients receiving their care at home, the principles of PC must also traverse the boundaries of the health care system.

\section{Funding Information}

No funding was received to support this work.

\section{Author Disclosure Statement}

No competing financial interests exist.

\section{References}

1. Morrison RS, Augustin R, Souvanna P, Meier DE: America's care of serious illness: A state-by-state report card on access to palliative care in our nation's hospitals. J Palliat Med 2011;14:1094-1096.

2. Kamal AH, Wolf SP, Troy J, et al.: Policy changes key to promoting sustainability and growth of the specialty palliative care workforce. Health Aff (Millwood) 2019;38:910-918.

3. Schmittdiel JA, Gopalan A, Lin MW, et al.: Population health management for diabetes: Health care system-level approaches for improving quality and addressing disparities. Curr Diab Rep 2017;17:31.

4. Smith G, Bernacki R, Block SD: The role of palliative care in population management and accountable care organizations. J Palliat Med 2015; 18:486-494.

5. Gupta R, Roh L, Lee C, et al.: The population health value framework: Creating value by reducing costs of care for patient subpopulations with chronic conditions. Acad Med 2019;94:1337-1342.

6. Bleser WK, Saunders RS, Winfield L, et al.: ACO serious illness care: Survey and case studies depict current challenges and future opportunities. Health Aff (Millwood) 2019;38:1011-1020.

7. Bodenheimer $\mathrm{T}$, Wagner $\mathrm{EH}$, Grumbach $\mathrm{K}$ : Improving primary care for patients with chronic illness: The chronic care model, Part 2. JAMA 2002;288:1909-1914.

8. Benton D, Ralls JD, Stern S, et al.: Optimizing. Specialty Palliative Care. In: Presented at: Center to Advance Palliative Care National Seminar; November 9-11, 2017; Phoenix, AZ, 2017.

9. Lakin JR, Koritsanszky LA, Cunningham R, et al.: A systematic intervention to improve serious illness communication in primary care. Health Aff (Millwood) 2017;36:1258-1264.

10. Compton-Phillips A, Mohta NS: The Power of Palliative Care: https://catalyst.nejm.org/power-palliative-end-of-lifecare-program/ (Last accessed October 7, 2019).

11. Kozlov E, Cai A, Sirey JA, et al.: Identifying palliative care needs among older adults in nonclinical settings. Am J Hosp Palliat Care 2018;35:1477-1482. 
12. Trivedi N, Peterson EB, Ellis EM, et al.: Awareness of palliative care among a nationally representative sample of U.S. adults. J Palliat Med 2019;22:1578-1582.

13. Kamal AH, Wolf S, Nicolla JM, et al.: Usability of PCforMe in patients with advanced cancer referred to outpatient palliative care: Results of a randomized, active-controlled pilot trial. J Pain Symptom Manage 2019;58:382-389.

14. Advance Care Planning (ACP) Decisions: https://acpdeci sions.org (Last accessed October 7, 2019).

15. PREPARE for Your Care: https://prepareforyourcare.org (Last accessed October 7, 2019).

16. Sudore RL, Boscardin J, Feuz MA, et al.: Effect of the PREPARE website vs an easy-to-read advance directive on advance care planning documentation and engagement among veterans: A randomized clinical trial. JAMA Intern Med 2017;177:1102-1109.

17. El-Jawahri A, Paasche-Orlow MK, Matlock D, et al.: Randomized, controlled trial of an advance care planning video decision support tool for patients with advanced heart failure. Circulation 2016;134:52-60.

18. Casarett D, Teno J: Why population health and palliative care need each other. JAMA 2016;316:27-28.

19. Build a Before I Die Wall: https://beforeidieproject.com/ participate (Last accessed October 7, 2019).

20. Dying to Know Day: https://www.dyingtoknowday.org/ planning (Last accessed October 7, 2019).

21. GW Cancer Care Palliative Care Awareness Toolkit: https://smhs.gwu.edu/cancercontroltap/sites/cancercontrol tap/files/Palliative\%20Care\%20Social\%20MediaToolkit\% 202018\%20FINAL.pdf (Last accessed October 7, 2019).

22. Freeman HP: The history, principles, and future of patient navigation: Commentary. Semin Oncol Nurs 2013;29:72-75.

23. Fischer SM, Kline DM, Min SJ, et al.: Effect of apoyo con carino (support with caring) trial of a patient navigator intervention to improve palliative care outcomes for latino adults with advanced cancer: A randomized clinical trial. JAMA Oncol 2018;4:1736-1741.

24. Mack JW, Weeks JC, Wright AA, et al.: End-of-life discussions, goal attainment, and distress at the end of life: Predictors and outcomes of receipt of care consistent with preferences. J Clin Oncol 2010;28:1203-1208.

25. Wright AA, Zhang B, Ray A, et al.: Associations between end-of-life discussions, patient mental health, medical care near death, and caregiver bereavement adjustment. JAMA 2008;300:1665-1673.

26. Wendler D, Rid A: Systematic review: The effect on surrogates of making treatment decisions for others. Ann Intern Med 2011;154:336-346.

27. Zhang B, Wright AA, Huskamp HA, et al.: Health care costs in the last week of life: Associations with end-of-life conversations. Arch Intern Med 2009;169:480-488.

28. Serious Illness Care: https://www.ariadnelabs.org/areas-ofwork/serious-illness-care/research/ (last accessed October 18, 2019).

29. Lakin JR, Block SD, Billings JA, et al.: Improving communication about serious illness in primary care: A review. JAMA Intern Med 2016;176:1380-1387.

30. VitalTalk: https://www.vitaltalk.org (last accessed October 18, 2019).

31. Baile WF, Buckman R, Lenzi R, et al.: SPIKES-A six-step protocol for delivering bad news: Application to the patient with cancer. Oncologist 2000;5:302-311.

32. Kruser JM, Taylor LJ, Campbell TC, et al.: "Best case/ worst case": Training surgeons to use a novel communi- cation tool for high-risk acute surgical problems. J Pain Symptom Manage 2017;53:711.e715-719.e715.

33. Stover AM, Tompkins Stricker C, Hammelef K, et al.: Using stakeholder engagement to overcome barriers to implementing patient-reported outcomes (PROs) in cancer care delivery: Approaches from 3 prospective studies. Med Care 2019;57(Suppl. 5, Suppl. 1):S92-S99.

34. Wysham NG, Cox CE, Wolf SP, Kamal AH: Symptom burden of chronic lung disease compared with lung cancer at time of referral for palliative care consultation. Ann Am Thorac Soc 2015;12:1294-1301.

35. Kamal AH, Bowman B, Ritchie CS: Identifying palliative care champions to promote high-quality care to those with serious illness. J Am Geriatr Soc 2019;67(S2):S461-S467.

36. Reidy J, Halvorson J, Makowski S, et al.: Health system advance care planning culture change for high-risk patients: The promise and challenges of engaging providers, patients, and families in systematic advance care planning. J Palliat Med 2017;20:388-394.

37. Rocque GB, Partridge EE, Pisu M, et al.: The patient care connect program: Transforming health care through lay navigation. J Oncol Pract 2016;12:e633-e642.

38. Rocque GB, Dionne-Odom JN, Sylvia Huang $\mathrm{CH}$, et al.: Implementation and impact of patient lay navigator-led advance care planning conversations. J Pain Symptom Manage 2017;53:682-692.

39. Molloy DW, Guyatt GH, Russo R, et al.: Systematic implementation of an advance directive program in nursing homes: A randomized controlled trial. JAMA 2000;283:1437-1444.

40. Black K, Fauske J: Exploring influences on community-based case managers' advance care planning practices: Facilitators or barriers? Home Health Care Serv Q 2007;26:41-58.

41. Fischer S, Min SJ, Cervantes L, Kutner J: Where do you want to spend your last days of life? Low concordance between preferred and actual site of death among hospitalized adults. J Hosp Med 2013;8:178-183.

42. ElMokhallalati Y, Mulvey MR, Bennett MI: Interventions to support self-management in cancer pain. Pain Rep 2018;3: e690.

43. Mayer DK, Travers D, Wyss A, et al.: Why do patients with cancer visit emergency departments? Results of a 2008 population study in North Carolina. J Clin Oncol 2011;29: 2683-2688.

44. Shepherd-Banigan M, Kaufman BG, Decosimo K, et al.: Adaptation and implementation of a family caregiver skills training program: From single site RCT to multisite pragmatic intervention. J Nurs Scholarsh 2019;52:23-33.

45. Van Houtven $\mathrm{CH}$, Smith VA, Lindquist JH, et al.: Family caregiver skills training to improve experiences of care: A randomized clinical trial. J Gen Intern Med 2019;34:2114-2122.

46. Everplans: https://www.everplans.com/advance-directive (Last accessed October 7, 2019).

47. MyDirectives: https://mydirectives.com (Last accessed October 7, 2019).

48. Cake: https://www.joincake.com (Last accessed October 7, 2019).

Address correspondence to: Devon K. Check, PhD Department of Population Health Sciences Duke Cancer Institute Duke University Medical Center Durham, NC

E-mail:devon.check@duke.edu 